\title{
DIABETES
}

\section{How does leptin decrease hyperglycaemia in T1DM and T2DM?}

A study published in Nature Medicine demonstrates that leptin suppresses the hypothalamic-pituitary-adrenal (HPA) axis in rat models of poorly controlled type 1 diabetes mellitus (T1DM) and type 2 diabetes mellitus (T2DM), resulting in decreased hepatic gluconeogenesis.

"Previous studies have shown that leptin can reduce plasma glucose concentrations in rodent models of T1DM in the absence of insulin and we were interested in understanding the mechanism," explains Gerald Shulman.

The researchers used novel NMR spectroscopy techniques to quantify liverspecific rates of hepatic gluconeogenesis and substrate oxidation in rat models of poorly controlled T1DM and T2DM. Stable isotope infusion studies were used to assess the rates of whole-body turnover of fatty acids, glycerol and acetate.

The analyses revealed that leptin decreased fasting plasma concentrations of glucose by inhibiting the HPA axis, which normalized plasma levels of corticosterone. This normalization of corticosterone levels led to reduced lipolysis, which resulted in decreased rates of hepatic glucose production via reductions in the rates of conversion of pyruvate and glycerol to glucose. Importantly, these effects could be dissociated from changes in plasma levels of insulin and glucagon. These findings suggest that lipolysis, secondary to hypoleptinaemia and increased HPA activity, has a critical role in hyperglycaemia.

"We would like to translate these findings to patients with poorly controlled T1DM and T2DM using novel ${ }^{13} \mathrm{C}$ NMR spectroscopy methods that we are developing," says Shulman.

Claire Greenhill

Original article Perry, R. L. et al. Leptin reverses diabetes by suppression of the hypothalamic-pituitary-adrenal axis. Nat. Med. doi:10.1038/nm.3579 